\title{
Erratum to: One-Step Synthesis of 2-[(2-Carboxyphenyl)amino]- 6-formylnicotinic Acid via Photolysis of 2-Azidobenzoic Acid in the Presence of Weak Bases
}

\author{
A. V. Budruev ${ }^{a}, *$, D. Yu. Dzhons ${ }^{b}$, M. A. Giricheva ${ }^{a}$, D. A. Davydov ${ }^{a}$, and A. V. Pokrovskaia ${ }^{a}$ \\ ${ }^{a}$ National Research Lobachevsky State University of Nizhny Novgorod, Nizhny Novgorod, 603950 Russia \\ ${ }^{b}$ Reper-NN Ltd., Nizhny Novgorod, 603003 Russia \\ *e-mail:budruev@gmail.com
}

Received December 2, 2021; revised December 3, 2021; accepted December 3, 2021

DOI: $10.1134 / \mathrm{S} 1070363221110219$

Supplementary material was replaced.

\section{SUPPLEMENTARY INFORMATION}

The online version contains supplementary material available at https://doi.org/10.1134/S1070363221110219

The original article can be found online at https://doi.org/10.1134/S1070363221100133 